\title{
SARCÓFAGOS ROMANOS DECORADOS DEL SIGLO IV EN EL TERRITORIO ANDALUZ: ENFOQUES Y PROBLEMÁTICA VIGENTE
}

\author{
FOURTH CENTURY BC ROMAN SARCOPHAGI IN ANDALUSIAN \\ TERRITORY: APPROACHES AND CURRENT ISSUES
}

\author{
MIGUEL ÁNGEL GARCÍA GARCÍA*
}

\begin{abstract}
Resumen: En 1971 Manuel Bendala comenzó su actividad investigadora publicando dos nuevos fragmentos de sarcófago, uniéndose al conjunto de estudios que han configurado el actual conocimiento de los sarcófagos decorados romanos en el antiguo territorio bético. Los sarcófagos publicados enmarcan cronológicamente el siglo IV, periodo caracterizado por la historiografía tradicional por dos grandes hitos: el paso de las producciones paganas a las producciones cristianas y el absoluto monopolio de las importaciones procedentes de los talleres metropolitanos. A este respecto, lejos de la aparente homogeneidad del conjunto, el actual estado de la cuestión obliga a reconsiderar algunas de las teorías vigentes y plantear nuevas soluciones a las dudas generadas por el carácter excepcional de algunas piezas.
\end{abstract}

Palabras clave: Sarcófagos, Antiguedad tardía, Importación, Iconografía, Métodos de producción.

A comienzos de la década de los setenta, Manuel Bendala publicó dos nuevos fragmentos de sarcófagos decorados andaluces (Bendala 1971). Este trabajo venía a sumarse al nutrido número de estudios que, desde la publicación del sarcófago de Écija (Salés y Ferré 1887; Fita 1887; Ficker 1887), se habían interesado por la presencia de estos objetos en el territorio, mientras que, de forma contemporánea, Manuel Sotomayor desarrollaba sus fundamentales investigaciones sobre los sarcófagos romanos con decoración cristiana en la

\footnotetext{
* Grupo de investigación HUM-702 (Universidad de Sevilla)
}

\begin{abstract}
In 1971 Manuel Bendala began its research publishing two new fragments of sarcophagus, thus joining the set of studies that have shaped the current understanding of Roman decorated sarcophagi in the former territory of Baetica. The sarcophagi published framed chronologically the fourth century, a period characterized by the traditional historiography of two major milestones: the passage of the pagan productions to christian productions and the absolute monopoly of imports from the metropolitan workshops. In this respect, far from the apparent homogeneity of the whole, the current state of the art necessary to reconsider some of the existing theories and propose new solutions to the doubts generated by the exceptional nature of some pieces.
\end{abstract}

Key words: Sarcophagi, Late Antiquity, Importation, Iconography, Production methods.

Península (Sotomayor 1973 y 1975). La publicación en 2006 del volumen del CSIR correspondiente a los sarcófagos romanos andaluces ha servido para actualizar y sistematizar el conocimiento sobre esta producción romana en Andalucía (CSIR I, 3) ${ }^{1}$.

De forma paralela al ingente esfuerzo desplegado por los investigadores para llevar a cabo la sistematización del conjunto conocido de sarcófagos romanos

1. Con posterioridad a este trabajo no ha habido aportaciones al conjunto a excepción de la identificación como sarcófago de una pieza anteriormente catalogada como relieve ibérico en el Museo Arqueológico y Etnológico de Córdoba (Sánchez Velasco 2008). 
mediante su catalogación, desde parámetros cronológicos y estilísticos, y la identificación de los motivos iconográficos representados, se han ido desarrollando estudios en los que se planteaban problemas asociados a esta importante producción de la cultura material romana $^{2}$. De este modo, durante las últimas décadas la investigación sobre los sarcófagos romanos ha ido progresivamente centrándose no tanto ya en el estudio individual de los ejemplares, sino en la ampliación de conocimiento sobre los procesos de producción, adquisición, transporte y utilización de los mismos, así como en la relación existente entre los clientes y los talleres. Estas nuevas líneas de investigación se presentan en la actualidad como la principal vía para avanzar en la resolución de algunas importantes problemáticas que aún son objeto de abierto debate.

Los dos fragmentos de sarcófago publicados por Bendala enmarcan cronológicamente un periodo que la historiografía tradicional ha caracterizado por dos grandes fenómenos: por un lado el paso de la producción de sarcófagos paganos a sarcófagos cristianos y por otro el absoluto dominio del comercio interprovincial por parte de los talleres metropolitanos. Estos argumentos, que gravitan en el análisis de los mencionados ejemplares, deben ser cuando menos matizados en el actual estado de la investigación.

\section{TALLERES Y CLIENTES ANTE LA INTRODUCCIÓN Y DIFUSIÓN DE LOS MOTIVOS CRISTIANOS EN LOS SARCÓFAGOS DECORADOS ROMANOS}

El más temprano de los fragmentos publicados por Bendala corresponde a una caja de sarcófago estrigilado conservada en una colección de la localidad sevillana de Lebrija y de la que se desconocen la procedencia y circunstancias precisas de hallazgo (CSIR I, 3, 64) (Fig.1). El fragmento conserva únicamente parte de uno de los registros escultóricos, ocupado por la figura de un pastor, igualmente de forma parcial uno de los campos de estrígiles y una mínima porción del campo central, donde puede apreciarse el arranque de un $p a-$ rapetasma. Las características formales de la pieza permiten datarla a finales del siglo III.

2. Esta labor ha sido desarrollada fundamentalmente por la investigación alemana a través de los sucesivos volúmenes de los corpora Die antiken Sarkophag-Reliefs (ASR) y Repertorium der christlich-antiken Sarkophage.

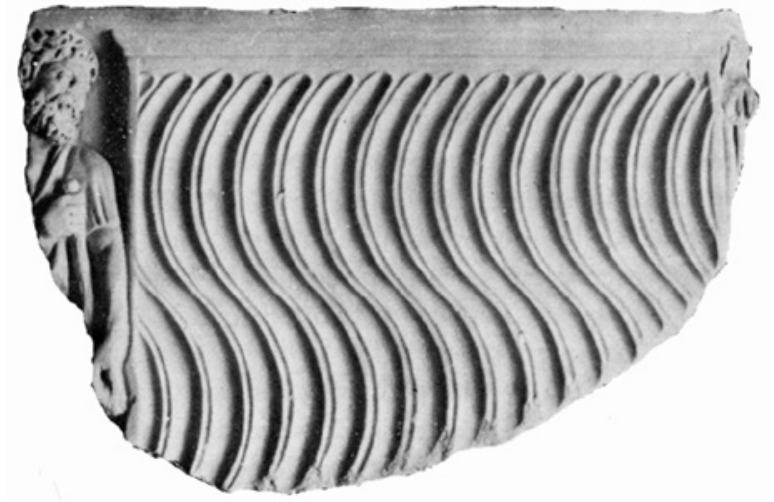

Figura 1. Sarcófago de Lebrija (CSIR I, 3, 64) (Fotografía Bendala, 1971)

Tipológicamente el fragmento sevillano puede identificarse con el tipo 12 de la clasificación de KochSichtermann (Koch y Sichterman 1982: 75). Este tipo de sarcófago estrigilado es uno de los más extendidos dentro de la producción romana de sarcófagos decorados, encontrándose igualmente bien representada en el territorio andaluz (Rodríguez Oliva 2002). Iconográficamente, el ejemplar se incluye dentro de un conjunto de sarcófagos que tuvo su mayor difusión entre finales del siglo III y comienzos del siglo IV y que se caracterizan por utilizar en sus programas decorativos motivos pastorales y de vida privada. La problemática historiográfica en torno a este conjunto de sarcófagos se ha centrado fundamentalmente en su adscripción como piezas paganas o cristianas. Este interés por su identificación ha llevado a los investigadores a catalogar estos ejemplares bajo un amplio número de denominaciones, como sarcófagos proto o criptocristianos, sarcófagos con decoración neutra, sarcófagos bucólicos o sarcófagos paradisíacos, amén de considerarlos únicamente sarcófagos paganos.

El debate en torno a la naturaleza de este grupo de sarcófagos plantea uno de los grandes problemas que la actual investigación sobre los sarcófagos romanos tardoantiguos debe solventar, como es la división excesivamente inflexible planteada por la historiografía tradicional entre sarcófagos cristianos y paganos. Esta división, que resulta correcta desde el punto de vista de la clasificación iconográfica, ha llevado sin embargo a arrastrar una serie de planteamientos previos poco acertados, y ha desembocado en el establecimiento de dos campos de investigación independientes y estancos, que en muchas ocasiones han ignorado mutuamente los avances realizados. En este sentido, y dentro del estado 
actual de la investigación, cada vez resulta más patente que la producción y utilización de sarcófagos decorados durante el periodo romano debe ser considerada ante todo un fenómeno completamente unitario, que se desarrolló de forma continuada desde el siglo II d.C. y durante toda la tardoantigüedad, sobreviviendo, como arraigada seña de identidad de la cultura romana, a la propia estructura política del Imperio.

Desde esta perspectiva, al igual que ocurre con otras producciones romanas tardoantiguas, la introducción de motivos cristianos en los repertorios decorativos no permite plantear argumentos de peso para considerar una ruptura en el funcionamiento de las officinae que hasta la fecha los venían produciendo ${ }^{3}$.

El origen de esta problemática división se encuentra sin duda en la sobrevaloración, por parte de la historiografía tradicional, del carácter excluyente de la religión cristiana. Así, el idealizado retrato del cristiano en consonancia con los escritos de los apologetas resulta más que matizable y plantea muchas incoherencias respecto a los mecanismos de producción y uso de la cultura material tardoantigua 4 . En el caso particular de los talleres escultóricos, esta argumentación tradicional desemboca en importantes interrogantes, como el posible papel intervencionista que las jerarquías eclesiásticas hubieron de asumir ante una hipotética interrupción programada de las producciones paganas. Como argumento alternativo a este problema, en términos generales, es posible concluir que la cristianización de los repertorios decorativos no tuvo que suponer una paralela cristianización de los talleres, y que fueron únicamente las relaciones entre oferta y demanda las que motivaron los cambios acaecidos en la producción de sarcófagos romana en el paso de los siglos III al IV. Para defender este argumento es necesario ante todo analizar la naturaleza y características de los clientes de este tipo de objetos y las características de su producción.

Los sarcófagos decorados fueron un producto de lujo destinado exclusivamente a los miembros de las

3. Un ejemplo significativo de esta continuidad es la producción de TSC norteafricana con decoración de relieve aplicado donde conviven indistintamente y de forma contemporánea motivos de naturaleza pagana y cristiana producidos con toda probabilidad por los mismos talleres (Bejaoui 1997).

4. Algunos casos significativos ilustran perfectamente una flexibilidad contraria a esta suerte de integrismo cristiano en relación con el estudio concreto de los sarcófagos, como el caso de la tumba de Flavius Memorius, cristiano que llegó a ocupar el cargo de gobernador militar de Mauritania Tingitana y fue inhumado en la necrópolis de Alyscamps en la primera mitad del siglo IV, reutilizando un sarcófago del siglo II decorado con motivo de esfinges y centauros $(\mathrm{Gu}-$ yon 2001: 52). jerarquías sociales y económicas, ya que únicamente estas podían asumir el alto coste de fabricación y transporte de estos objetos. Los estudios prosopográficos realizados en base a la epigrafía presente en el conjunto de sarcófagos conocidos ratifican este hecho, arrojando el análisis del origen y profesiones de los usuarios de este conjunto un cierto nivel de diversidad social, pero en el que en líneas generales predominan los miembros de las élites urbanas y los militares de alto rango. La absoluta continuidad que este análisis presenta en las inscripciones posteriores tras la paz de la Iglesia respecto a la situación previa es patente y merece ser remarcada ${ }^{5}$.

Durante el siglo III estas jerarquías, al igual que el resto de la sociedad romana, se encuentran inmersas en un importante cambio de mentalidad en relación con el mundo funerario, fundamentalmente debido al auge de las religiones de tipo salvífico, entre ellas el cristianismo. En el campo concreto de la utilización de sarcófagos decorados esta nueva percepción del más allá se va a traducir en un progresivo abandono de los grandes temas y ciclos mitológicos en favor de la utilización de programas iconográficos basados tanto en aspectos de la vida cotidiana como en la representación del locus amoenus (Ewald 2003). Estos cambios no van a mermar, sin embargo, las necesidades de autorrepresentación de estas jerarquías tardoantiguas que, por el contrario, se muestran especialmente sensibles ante este aspecto. En este sentido, la crisis del evergetismo clásico no hizo sino concentrar los esfuerzos de representación de estas élites en el ámbito privado.

De esta forma, los talleres escultóricos van a mostrar durante los inicios del siglo IV un alto nivel de flexibilidad para modificar su producción ante las necesidades de sus clientes, del mismo modo que habían sido receptivos a los cambios de sensibilidad producidos durante el siglo III. En un primer momento el marcado carácter polisémico del repertorio de motivos del citado conjunto de sarcófagos permitió a estos talleres satisfacer la demanda de sus clientes tanto cristianos como seguidores de otras religiones ${ }^{6}$. Posteriormente

5. Como ejemplo significativo de este fenómeno puede destacarse que en el conjunto de sarcófagos conocidos se han documentado un total de 39 inscripciones senatoriales cristianas frente a 15 inscripciones senatoriales paganas (Desken-Weiland 2004: 152). En el mismo sentido, la adopción de ciertas costumbres como la utilización de nombres peyorativos entre los cristianos, que se documenta en los enterramientos comunes de las catacumbas metropolitanas (Kajanto 1962), contrasta con el mantenimiento en la utilización de los tria nomina en la epigrafía sarcofágica cristiana.

6. Desde un punto de vista iconográfico parece claro que este conjunto de piezas no puede identificarse inequívocamente con el cristianismo, aunque tampoco pueden ser consideradas, en stricto 
y probablemente ante un proceso generalizado de cristianización de las jerarquías sociales, comienzan a introducirse progresivamente motivos cristianos en la producción de sarcófagos romanos. En esta evolución resulta fundamental tener en cuenta las características de los mecanismos de producción para comprender correctamente este proceso. Así, es posible identificar dos vías en la introducción de estos motivos. Por una parte los talleres comienzan a integrarlos en los esquemas compositivos previos, lo que sin duda permitía mantener una producción en serie sin alterar significativamente los procesos de fabricación. Buen ejemplo de este procedimiento es un sarcófago estrigilado del $\mathrm{Mu}-$ seo Nazionale de Roma (Rep. 1, 777) que presente en su frente el mismo programa decorativo que el fragmento de Lebrija y cuyos laterales se decoran con el motivo cristiano del bautismo de Cristo y un grupo de corderos dispuestos en registros. En otros casos los motivos cristianos van a situarse en los áticos de las tapaderas, como es el caso del sarcófago de Osimo (Rep. 2, 185), en el que los motivos de la adoración de los Magos y el ciclo de Jonás se sitúan en el ático mientras que en el frente de la caja se desarrolla un motivo de caza $^{7}$. Por otro lado comienzan a desarrollarse casi experimentalmente nuevas soluciones formales en las que se introducen los motivos cristianos, como en el caso del sarcófago de Veletri (Rep. 2, 242). De nuevo, una lectura a partir de los procesos productivos permite explicar la posible razón por el que este tipo de esquemas no tendrán continuidad en el tiempo, dado que la complejidad que suponía la composición de motivos superpuestos en un campo abierto no se adaptaba sin duda bien a los sistemas de fabricación en serie.

En este contexto, la progresiva desaparición de los motivos iconográficos paganos en la producción sarcofágica del siglo IV debe ser explicada más allá de un rechazo religioso por parte de un cristianismo cada vez más extendido, ya que estos temas continúan presentes en otras producciones de la cultura material romana contemporánea, como es el caso de la musivaria, tanto

sensu, como piezas exclusivamente paganas. La descontextualización casi absoluta del conjunto de ejemplares conocidos es, sin lugar a dudas, uno de los principales escollos que debe salvar la investigación al respecto. De este modo, el conocimiento de los contextos posibilitaría ratificar la utilización generalizada de estos sarcófagos por parte de cristianos o paganos, como parece deducirse de algunos casos concretos en los que los contextos si permiten plantear este argumento (Bisconti 2004: 57).

7. Los evidentes paralelos de este ejemplar con el sarcófago de caza del Musée de 1'Arles Antique (ASR I, 2, 3), que no incorpora motivos cristianos, plantean una producción seriada de sarcófagos de este tipo durante todo el primer tercio del siglo IV. en ámbitos privados como incluso en edificios de culto cristiano, la orfebrería o la producción textil ${ }^{8}$. No obstante, es necesario matizar que los temas paganos van a continuar utilizándose durante gran parte del siglo IV en la producción sarcofágica, aunque ubicados una posición secundaria dentro de las decoraciones arquitectónicas, incluyéndose en pequeñas escenas en los áticos de las tapaderas u ocupando los laterales de las cajas, como había ocurrido inicialmente con los temas cristianos. Un buen ejemplo de este fenómeno es el famoso sarcófago de Junio Basso (Rep. 1, 680), cuyos laterales presentan una rica decoración con motivos de erotes que vendimian, siegan y caracterizan las estaciones.

La especialización por parte de los talleres en una producción de sarcófagos con decoración de temática cristiana es sin duda fruto de una demanda mayoritaria de estos objetos. Sin embargo, la gran duda que plantea este hecho es la continuidad en la utilización del sarcófago como enterramiento privilegiado por parte de la aristocracia pagana, que durante todo el siglo IV sigue estando presente y pujante en la sociedad romana (Brandenburg, 2002). Así, el radical descenso del número de sarcófagos paganos a partir del primer tercio del siglo IV es un fenómeno que aún no ha sido completamente resuelto, llegando incluso a plantearse un hipotético uso de sarcófagos con decoración cristiana por parte de estos miembros de las jerarquías romanas (Dresken-Weiland 2004: 152).

En el caso bético la presencia de sarcófagos paganos datados en el siglo IV se reduce hasta la fecha al ejemplar con motivo de aduentus de Madinat al-Zahra (CSIR I, 3, 21), que puede datarse ya en la segunda mitad del siglo (Fig. 2). La identificación de la pieza como obra de un taller local permite argumentar inicialmente, respecto a la problemática planteada, que mientras los grandes talleres centraban su producción en serie en la creación de sarcófagos con decoración cristiana, la creación por encargo directo hubo de representar la mejor forma de adquirir un sarcófago con una temática inusual ${ }^{9}$.

8. Este repertorio pagano tardío no está únicamente compuesto por motivos "neutros" sino que hace uso de los ciclos mitológicos que ya habían caído en desuso durante el siglo III en la decoración de sarcófagos. Así, es significativo como la utilización del mito como muestra de prestigio social va a ser restringida durante la tardoantigüedad a ámbitos bien definidos de la vida pública y privada.

9. Este argumento se plantea igualmente en el territorio peninsular en un grupo de sarcófagos con decoración pagana y posible origen común en un taller de Barcino, activo durante la primera mitad del siglo IV (CSIR II, 2, A1, A2, A3). 


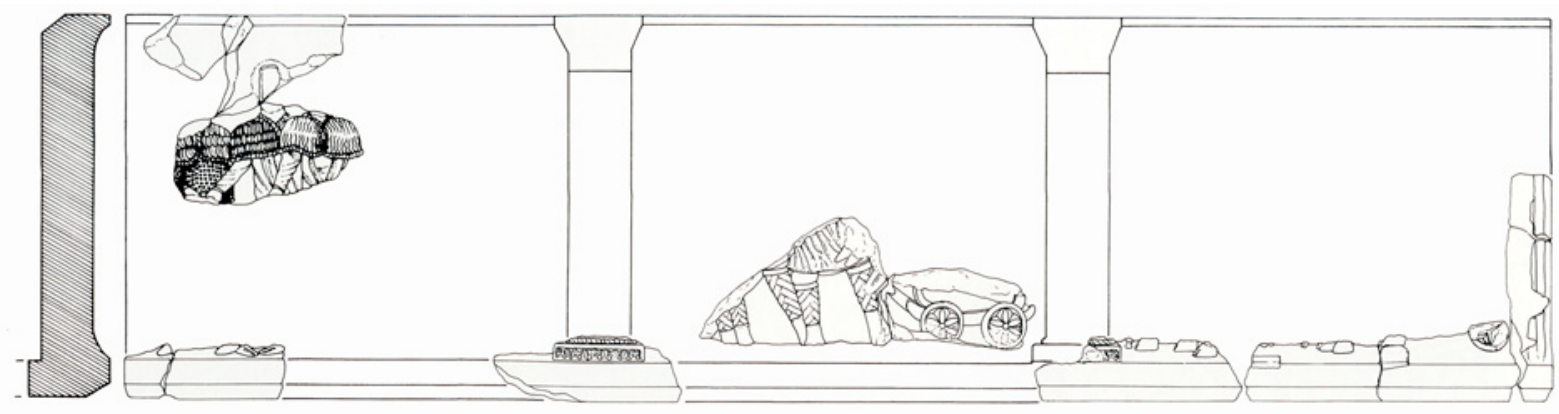

Figura 2. Sarcófago con escena de aduentus (CSIR I, 3, 21). Restitución según E. Conlin (Fotografía CSIR I, 3).

\section{PRODUCCIÓN EN SERIE, UNICA ICONOGRÁFICOS Y MATERIAS PRIMAS.}

Desde los trabajos pioneros de Ward-Perkins (WardPerkins 1975), diferentes autores han fundamentado sus estudios sobre los sarcófagos romanos bajo la premisa de su identificación como resultado de un sistema productivo en serie, llegando incluso a hablar de una industria de sarcófagos romana (Eichner 1981; Immerzeel 1996) ${ }^{10}$.

Resulta pertinente plantear que una producción en serie, o al menos una producción basada en la generación de stock, implicaría un cierto grado de estandarización en los productos, refiriéndonos tanto a las técnicas de fabricación como a los motivos iconográficos utilizados. En el ámbito bético, el sarcófago de Martos (CSIR I, 3, 58) puede ser considerado un buen ejemplo de este tipo de ejemplares producidos en serie durante el siglo IV (Fig. 3). En este sentido, y asumiendo una cierta flexibilidad en el desarrollo de variaciones sobre los motivos y esquemas utilizados, así como los propios procesos de creación y evolución iconográfica, una cuestión que este argumento deja abierta es la presencia de unica iconográficos. Sobre este aspecto, y considerando el nivel actual de sistematización y documentación del conjunto conocido, resulta cada vez más evidente que la consideración de estas excepcionalidades puede aportar claves significativas para la ampliación del conocimiento de estas producciones.

Algunos argumentos posibles permiten explicar la presencia de un unicum iconográfico. Por un lado, y de forma opuesta a los presupuestos de una producción

10. Efectivamente, algunas de las características presentes en la producción de sarcófagos, como son la organización jerarquizada del trabajo, la utilización de materias primas semielaboradas, la producción de stock o la estandarización de modelos, parecen adecuarse, con las cautelas necesarias, a unas condiciones preindustriales (Russell 2011: 121). basada en la generación de stock, la fabricación de un objeto bajo encargo directo, en el que el cliente tendría un cierto nivel de elección de los motivos, al modo de la práctica de la ordinatio epigráfica, posibilitaría la aparición de estos unica. Este tipo de producción bajo encargo y la diversidad iconográfica que posibilita parece corresponder ampliamente con la problemática planteada por la existencia de talleres locales frente a los grandes talleres especializados del entorno metropolitano.

En el ámbito concreto de los sarcófagos béticos, esta problemática afecta directamente a la hipótesis tradicional que consideraba la totalidad de los ejemplares de sarcófagos con decoración cristiana datados en el siglo IV como productos de importación procedentes de los talleres romanos. Así, Koch ha propuesto la reconsideración de que el sarcófago de Berja (CSIR I, 3, 1) pudiera ser obra de un taller local, aun respondiendo por completo a las características formales de estos talleres metropolitanos, en base a la presencia en el programa decorativo de estas peculiaridades iconográficas de las cuales el unicum del motivo de Pedro y Pablo ante Nerón es la más patente (Koch, 2000, 186, 367, 525) (Fig. 4).

La convergencia de dos de las cuestiones planteadas como son la excepcionalidad iconográfica y la estandarización de los procesos productivos, permite arrojar información sobre la posible adscripción de piezas a talleres de origen y, en el caso concreto de los sarcófagos béticos, permite exponer nuevas hipótesis sobre los materiales importados del siglo IV. De este modo, considerando que la fabricación de estos objetos corresponde a un sistema organizado de trabajo de taller, es factible plantear que los motivos decorativos de carácter secundario fueran más sensibles a la estandarización, formando parte de esquemas compositivos prefijados sobre los que se desarrollan los motivos principales. Así, cuando esta decoración secundaria 


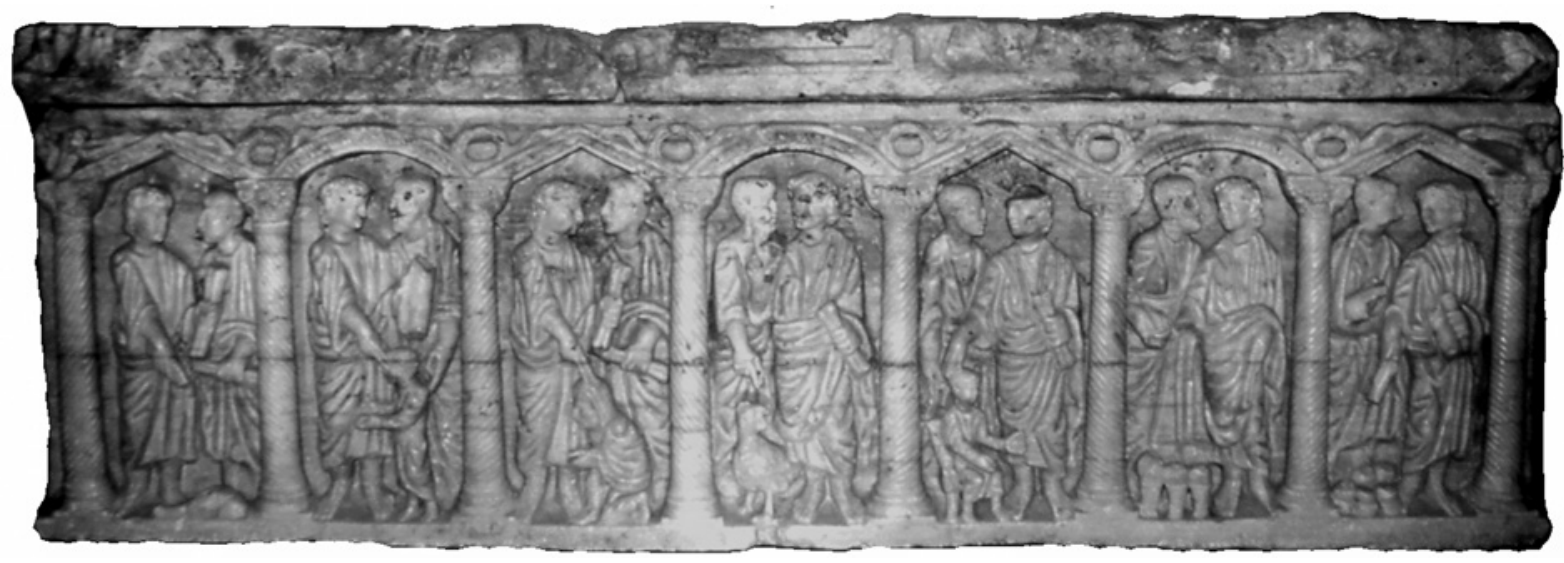

Figura 3. Sarcófago de Martos (CSIR I, 3, 58) (Fotografía del autor)

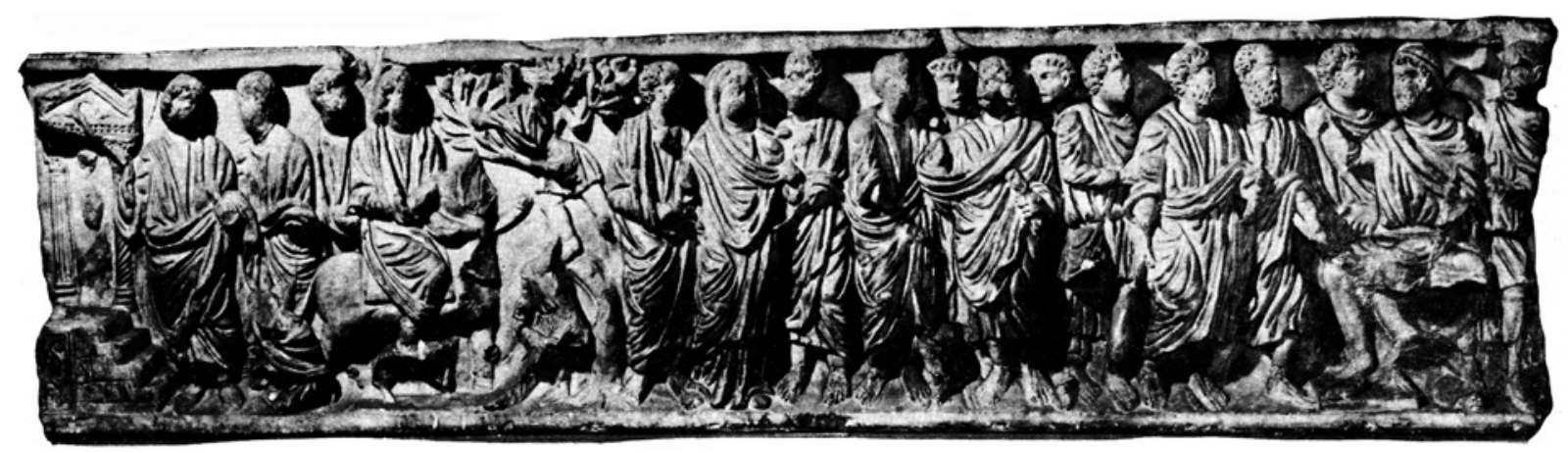

Figura 4. Sarcófago de Berja (CSIR I, 3, 1) (Fotografía DAI)

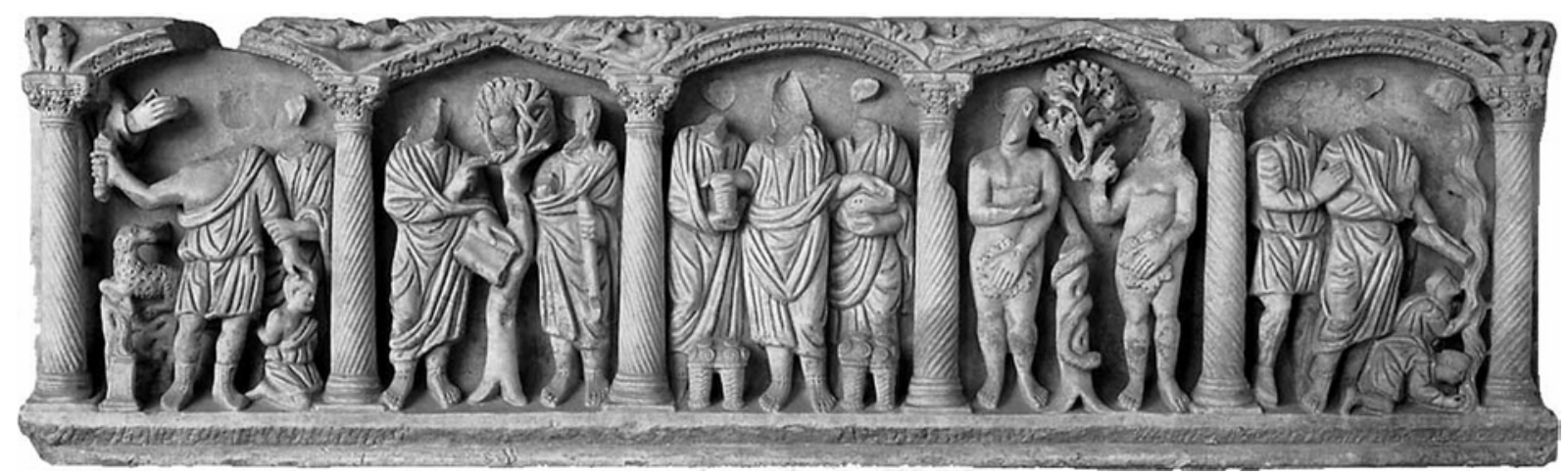

Figura 5. Sarcófago de Huerta de San Rafael (CSIR I, 3, 26) (Fotografía del autor)

presenta peculiaridades iconográficas significativas estas podrían ser consideradas como un indicador válido para la identificación de un taller.

Para la problemática bética, el ejemplo significativo de esta cuestión es la utilización de un motivo pagano, el tritón timonel que hace sonar una caracola, en la decoración secundaria de algunos sarcófagos del siglo IV. Este motivo aparece decorando las enjutas de los extremos en las cajas de los sarcófagos columnados béticos de Huerta de San Rafael (CSIR I, 3, 26) (Fig. 5) y Martos (CSIR I, 3, 58), así como posiblemente el sarcófago estrigilado con motivo arquitectónico central de la Ermita de los Mártires (CSIR I, 3, 25). Fuera del territorio bético el motivo está documentado en 


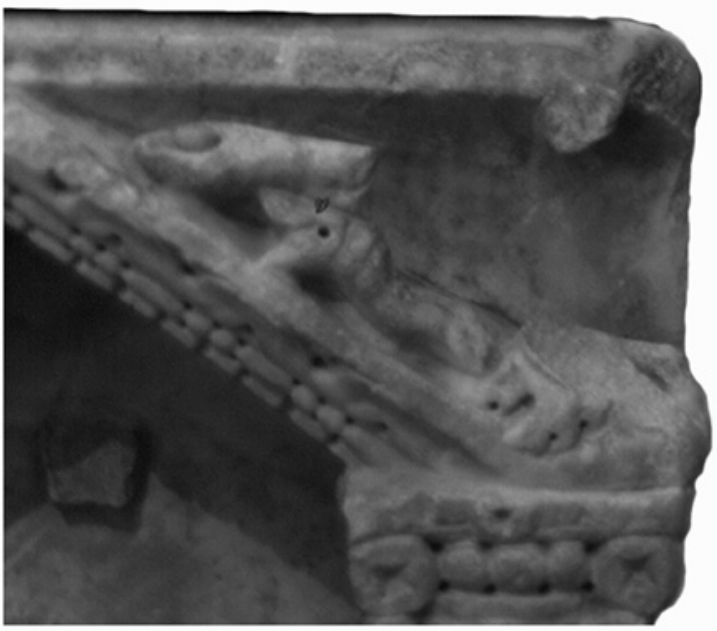

Figura 6. Sarcófago del Musée de l'Arles Antique (Rep. 3, 62). Detalle (Fotografía del autor)

producciones del siglo IV en el sarcófago columnado de Leyden (Rep. 2, 138), tres sarcófagos columnados de Arlés (Rep. 3, 53, 56, 62) (Figs. 6, 7), un sarcófago columnado de Nimes ( $W s, \mathrm{XVI}, 2)$ y un pequeño fragmento conservado en Ginebra (Rep. 2, 133). La total ausencia de paralelos conocidos en el conjunto metropolitano, que por otro lado supone la mayor parte del volumen total de ejemplares existentes hasta la fecha, es sin duda un hecho mucho más que relevante y permite lanzar la hipótesis del origen común de estas piezas en un taller provincial $^{11}$.

Otras relaciones en base a la presencia de particularidades iconográficas parecen ratificar igualmente este argumento. Por un lado la utilización, igualmente inédita en el ámbito romano, de algunos motivos del ciclo de Jonás en las enjutas de la decoración arquitectónica de las cajas de sarcófago de Huerta de San Rafael y Leyden, cuyas relaciones iconográficas y formales ya fueron puestas en evidencia por Sotomayor (Sotomayor, 1975, 121-127). La peculiar utilización de este motivo sirve asimismo para relacionar con el conjunto

11. La cronología de los ejemplares que componen este conjunto se sitúa entre el 330-340, datación de la caja de sarcófago de Huerta de San Rafael, y el 390-400, fecha a la que puede adscribirse el sarcófago de la Traditio Legis de Arlés (Rep. 3, 53). Se trataría, pues, de un taller con una actividad estable y prolongada en el tiempo durante la que se hace patente la evolución iconográfica del conjunto, en el que se desarrollan desde los motivo petrinos propios del periodo constantiniano, a los temas de pasión y esquemas esterotipados como la Traditio Legis que se desarrollarán desde finales del siglo IV y fundamentalmente en el siglo $\mathrm{V}$, todo ello manteniendo sin cambios significativos la decoración secundaria del marco arquitectónico.

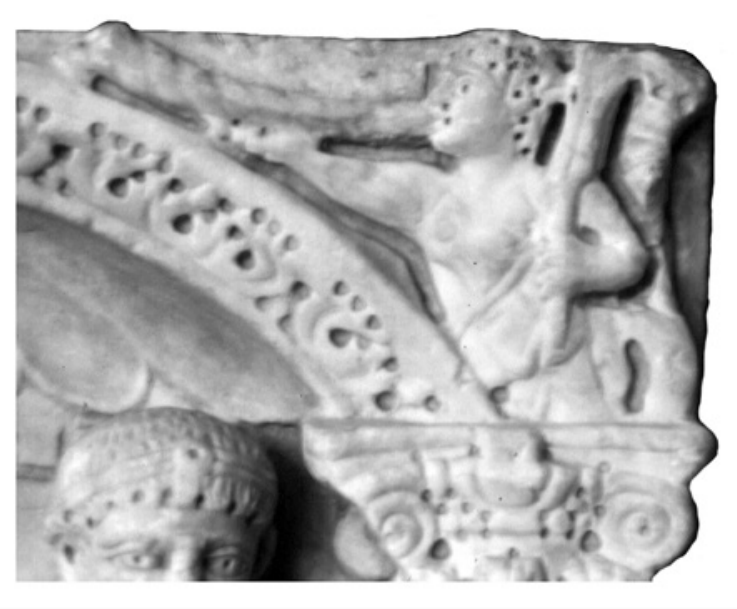

Figura 7. Sarcófago del Musée de l'Arles Antique (Rep. 3, 53). Detalle (Fotografía del autor)

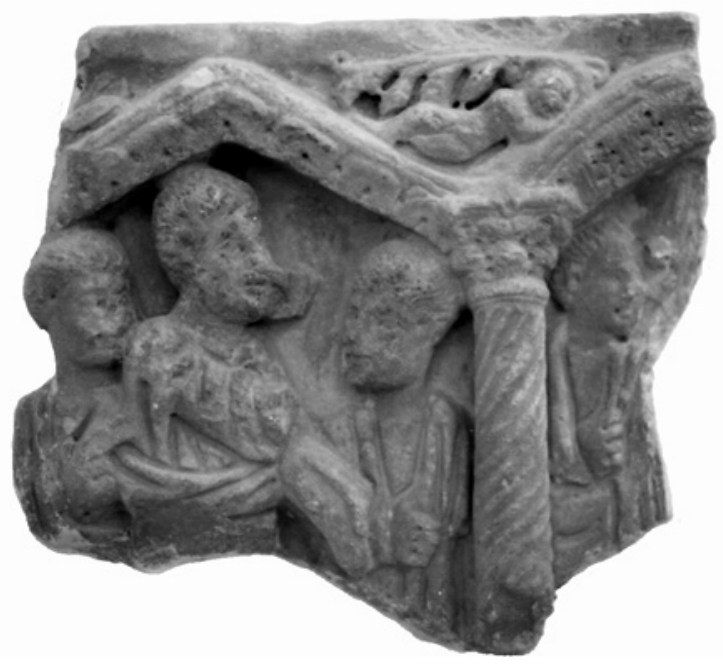

Figura 8. Sarcófago de Los Palacios (CSIR I, 3, 65) (Fotografía del autor)

mencionado otro ejemplar bético, el fragmento de sarcófago columnado de Los Palacios (CSIR I, 3, 65) (Fig. 8). Por otro lado, si se considera la estandarización de motivos dentro de los procesos de producción de los talleres, la evidente similitud entre la escena del sacrificio de Isaac en el primer intercolumnio del sarcófago de Huerta de San Rafael y la representada, aunque desgraciadamente en muy mal estado de conservación, en la misma posición en la caja de sarcófago columnado de Arlés (Rep. 3, 52), la hipótesis de un origen común adquiere aún mayor consistencia. 


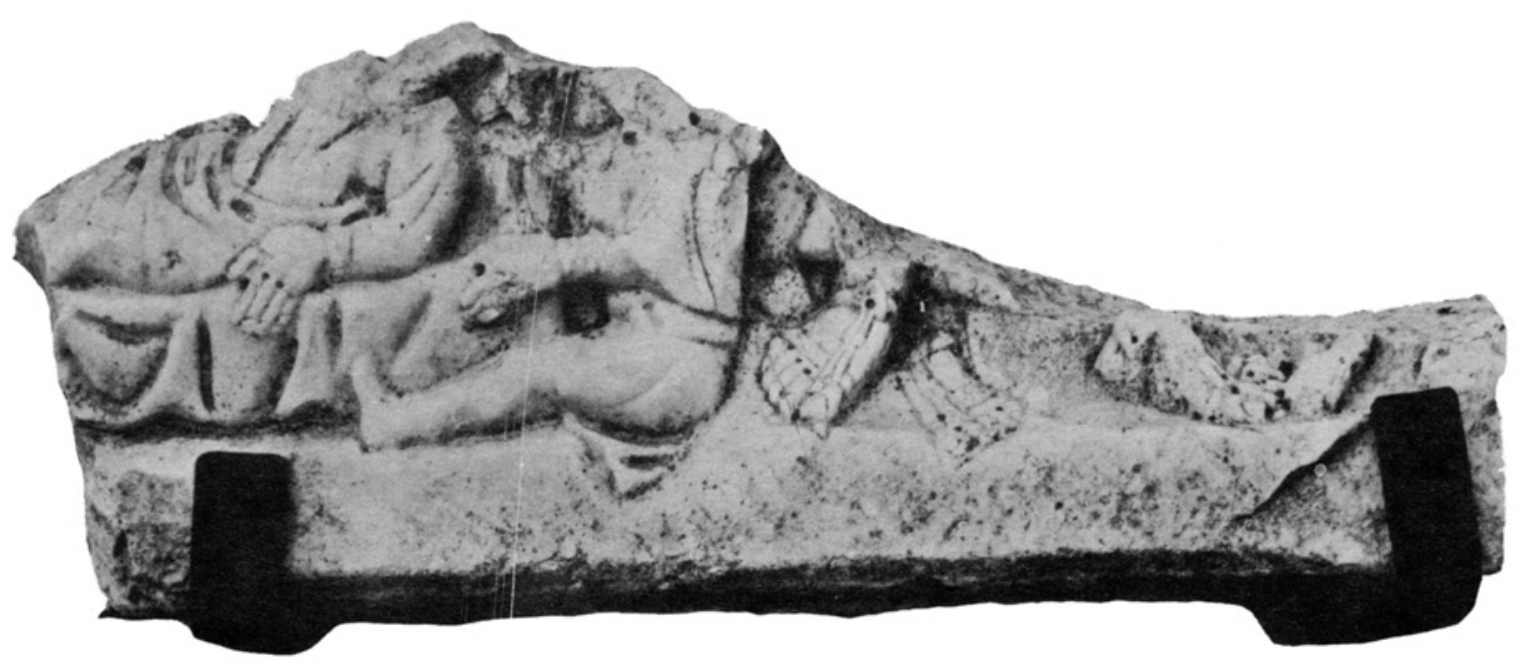

Figura 9. Sarcófago de la Peñuela (CSIR I, 3, 4) (Fotografía Sotomayor, 1975)

Las conclusiones que arrojan las relaciones existentes dentro de este conjunto ponen en entredicho la teoría tradicional, planteando no sólo que durante el siglo IV existen talleres locales activos que producen materiales del mismo nivel de calidad que elaborados en el foco metropolitano, sino que compiten con estos mismos productos en el mercado interprovincial ${ }^{12}$.

El debate sobre la deslocalización de los talleres de sarcófagos durante el siglo IV centra del mismo modo el análisis del segundo de los fragmentos publicados por Bendala, conservado en la finca La Peñuela de Jerez de la Frontera (CSIR I, 3, 4) (Fig. 9). Este corresponde a una serie bien identificada de sarcófagos, denominada por su programa iconográfico como sarcófagos de Bethesda (Nicoletti 1981). Esta serie está compuesta por un conjunto de ejemplares disperso por Gallia, Hispania, Roma y el norte de África, así como un ejemplar en la isla de Ischia. Sus similitudes obligan a pensar en que fueron productos surgidos de un mismo taller. En este sentido, el debate planteado por todos los investigadores ha sido la localización de este taller en el foco metropolitano, mayoritariamente aceptada, o por el contrario su ubicación

12. La existencia de un importante taller productor sudgálico durante el siglo IV ha sido objeto de un largo e intenso debate desde los trabajos de Benoit. Koch, tras llevar a cabo el estado de la cuestión (Koch, 2000, 471, 491), ha vuelto a proponer el carácter local de la mayor parte de los sarcófagos del siglo IV documentados en la Narbonense e incluso la capacidad exportadora de este foco gálico. Esta hipótesis difiere de la opinión de la mayor parte de los investigadores actuales (Caillet 1993; Immerzeel y Jongste 1993). en el entorno gálico ${ }^{13}$. En la resolución de esta cuestión vuelve a ser crucial el análisis de los procesos productivos. Mientras que la distribución geográfica de los ejemplares, mucho más abundantes en el territorio francés, y las características formales del conjunto pudieran hacer pensar en un posible origen galo, la excepcionalidad del ejemplar procedente de la catacumba del Pretestato (Rep. 1, 556) obliga a considerar el origen metropolitano de la serie. Este ejemplar, muy fragmentado, se encuentra parcialmente decorado en su mitad izquierda, mientras que la derecha está ocupada por una inscripción métrica en la que Gaudencio dedica la inscripción a su esposa difunta Bassa. Sobre esta inédita composición se ha argumentado que inscripción y decoración fueron contemporáneas, siguiendo un esquema intencionado (Mazzei 2004: 113114). Este argumento se lleva a cabo por la valoración del interés de la inscripción y la relación cronológica entre inscripción y decoración, aunque parece restar importancia al hecho de que la pieza se encuentra inacabada, como demuestra la falta de terminación tanto del campo donde se desarrolla la inscripción, como del relieve. Este extremo parece ratificar por un lado el origen metropolitano de la pieza, puesto que es poco probable el transporte de una pieza inacabada para un usuario final, y por otro plantea información sobre sobre las relaciones entre clientes y talleres. La posibilidad más factible es que Gaudencio hubiese encargado

13. Inicialmente se propusieron igualmente el origen microasiático (Lawrence 1927: 23-24) e incluso palestino (Simon 1938: 206). 
la pieza con anterioridad a la muerte de su esposa y quisiese disponer de ella antes de su finalización, puesto que resulta poco factible pensar que adquiriese una pieza incompleta. Ello implicaría, de otra parte, una ausencia de stock para un producto tan seriado como los sarcófagos de Bethesda ${ }^{14}$.

Por último, el análisis petrográfico de los materiales se ha mostrado en las últimas décadas como una herramienta útil para avanzar en toda esta investigación y dirimir alguno de los principales debates abiertos sobre el tema (Rodà 2001). No obstante, lejos de considerar este método como la última respuesta a las dudas planteadas, es necesario considerar las limitaciones técnicas que en la actualidad aún presenta este tipo de estudio. Por una parte el hecho de que casi la práctica totalidad del conjunto conocido se encuentre realizado en mármoles blancos complica el proceso de identificación del material. De otro lado, mientras que se dispone de un relativo buen conocimiento de las principales canteras explotadas durante el periodo romano, un gran número de canteras locales no han sido aún objeto de sistematización. Junto a estas limitaciones técnicas, otro de los obstáculos para la investigación es el escaso número de analíticas realizadas hasta la fecha, en comparación con el conjunto conocido de materiales. Así pues, la identificación de los mármoles de la mayor parte de los ejemplares publicados se encuentra determinada en base a observaciones de visu, lo que amplía la posibilidad de errores e imprecisiones durante el proceso.

En lo referente a los sarcófagos béticos, la historiografía ha identificado tradicionalmente la utilización de mármol con las producciones importadas desde el foco metropolitano, en contraposición con los ejemplares de taller local esculpidos en calizas. El estado actual de la investigación obliga a replantear la cuestión en base a algunas excepciones significativas. En primer lugar el análisis realizado por Ward-Perkins en 1970 a algunos de los fragmentos de sarcófagos béticos del siglo IV (Sotomayor 1975), arrojó como principal descubrimiento la identificación como posible mármol procedente de la cantera de Saint Béat, en los Pirineos, del material en el que se encuentra realizado el fragmento de sarcófago columnado de Los Palacios (CSIR I, 3, 65). Considerando las posibles limitaciones técnicas en cuanto la identificación de la cantera, lo que sí resulta razonable es la confirmación de que el material

14. El fenómeno de la utilización de piezas inacabadas, del que es ejemplo paradigmático el sarcófago de Portonaccio, lleva a otro campo de debate abierto, como es el de la visibilidad de las piezas en su contexto de utilización. no correspondía a los mármoles itálicos utilizados por los talleres romanos. Este hecho dejaría abierta dos posibilidades. En primer lugar es probable que la pieza sea obra de un taller local realizada sobre un mármol de cantera local. Por otro lado la ratificación en la identificación como mármol pirenaico dejaría abierta la posibilidad de una importación gálica, a lo que ya apunta el análisis de sus particularidades iconográficas.

Por otro lado, la pretendida adscripción exclusivamente foránea de las piezas por la utilización de mármol es cuestionable ante la sola presencia de algunos ejemplares en este material que, por sus características formales, pueden ser adscritos a talleres locales del siglo IV. Este es el caso de la caja de sarcófago con motivo de aduentus de Madinat al-Zahra (CSIR I, 3, 21) y el relieve con representación de Orfeo del Museo Arqueológico y Etnológico de Córdoba (CSIR I, 3, 74), que aunque no corresponde a un sarcófago, es un nuevo argumento para defender la presencia de un taller escultórico local con los medios suficientes para producir este tipo de materiales, aunque no asegura un abastecimiento estable por medio de la explotación de una cantera local.

Esta problemática desemboca en la consideración del fenómeno de los spolia y la reutilización de mármoles, común a partir del siglo III (Pensabene 1993). La reutilización de mármol para la fabricación de sarcófagos decorados ha sido defendida en base a la identificación de mármoles provenientes de canteras activas hasta el siglo II en algunos ejemplares del siglo IV dentro del ámbito metropolitano (Van Keuren et alii 2011: 179). Desde un punto de vista teórico resulta difícil plantear la posibilidad de que la reutilización de mármol, especialmente bloques arquitectónicos, fuera la fuente principal de aprovisionamiento para los talleres de sarcófagos metropolitanos durante todo el siglo IV, ya que el conjunto de ejemplares y los procesos de fabricación en serie que supone tal volumen de piezas implicaría la necesidad de un aprovisionamiento estable de material que únicamente la explotación de una cantera podría permitir. Sin embargo, abierta tal posibilidad con la confirmación del análisis detallado, una cuestión necesaria para el avance de la investigación es la consideración de que este mismo fenómeno de reutilización es válido para defender la existencia de talleres provinciales durante el siglo IV ${ }^{15}$.

15. Este argumento afectaría fundamentalmente al mencionado debate sobre el foco gálico, puesto que la crítica sobre su importancia ha estado en gran parte basada en la identificación de los mármoles utilizados. 


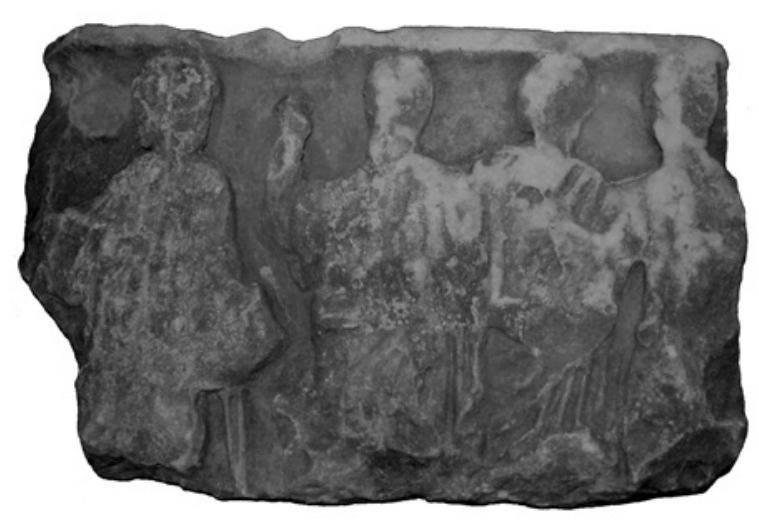

Figura 10. Sarcófago con decoración de friso continuo procedente de Córdoba (CSIR I, 3, 22) (Fotografía del autor)

Dentro del conjunto de materiales béticos un ejemplar pudiera relacionase con este fenómeno de reutilización. Se trata de un fragmento de frente de sarcófago procedente de una excavación arqueológica en la ciudad de Córdoba (CSIR I, 3, 22) (Fig. 10). El fragmento apareció reutilizado como umbral de puerta en una vivienda islámica. El borde superior del fragmento presenta una moldura cuyo origen pudiera encontrarse tanto en el reaprovechamiento de material arquitectónico para la elaboración del frente de sarcófago como en la reutilización durante el periodo hispanovisigodo de la caja de sarcófago como elemento arquitectónico (García García 2004: 246-248) (Fig. 11). La primera de estas posibilidades implica un ulterior problema, considerando que la naturaleza del hipotético elemento arquitectónico obliga a pensar que la pieza hubo de ser parte de un sarcófago ensamblado.

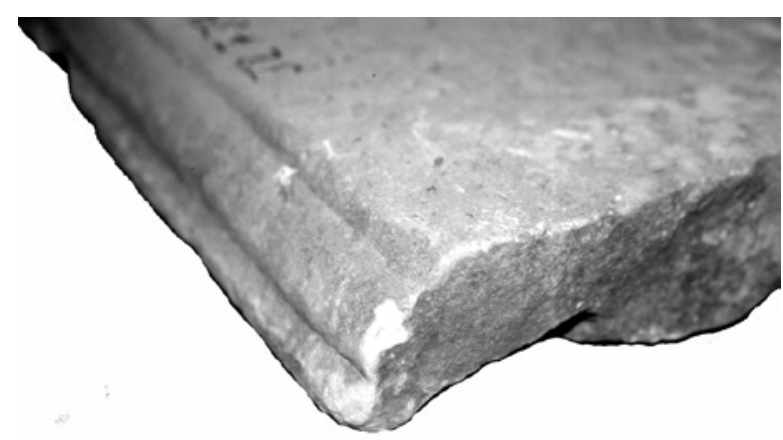

Figura 11. Sarcófago con decoración de friso continuo procedente de Córdoba (CSIR I, 3, 22). Detalle borde superior (Fotografía del autor)
Todos estos argumentos nos obligan a concluir que el conocimiento sobre la producción y utilización de sarcófagos decorados en la cultura romana, y en concreto de los sarcófagos andaluces, dista aún de ser completo. Así, los importantes pasos dados en cuanto a la sistematización y catalogación deben ser ampliados con el desarrollo de nuevas líneas de investigación, técnicas y metodologías. En este avance la consideración de las excepcionalidades presentes en el conjunto parecen perfilarse como la clave para redefinir muchas de las teorías vigentes, en las que el peso de ciertos prejuicios decimonónicos, como la identificación de los focos productores en base a una valoración de la calidad de las piezas, sigue encontrándose excesivamente presente.

\section{ABREVIATURAS}

ASR I, 2 = ANDREAE, B. (1980): Die römischen Jagdsarkophage. Berlin.

CSIR II, 2 = VIDAL, S. (2005): La escultura hispánica figurada de la Antigüedad tardía (Siglos IV-VII). Murcia

CSIR I, 3 = BELTRÁN FORTES, J., GARCÍA GARCÍA, M.A., RODRÍGUEZ OLIVA, P. (2006): Los sarcófagos romanos de Andalucía. Murcia.

Rep. 1 = BOVINI, G; BRANDENBURG, H. (1967): Repertorium der christlich antiken Sarkophage I. Rom und Ostia. Wiesbaden.

Rep. 2 = DRESKEN-WEILAND, J. (1998): Repertorium der christlich antiken Sarkophage, 2. Italien mit einem Nachtrag Rom und Ostia, Dalmatien, Museen der Welt. Mainz.

Rep. 3 = CHRISTERN-BRIESENICK, B. (2003): Repertorium der christlich antiken Sarkophage, 3. Frankreich Algerien Tunesien. Wiesbaden.

Ws = WILPERT, J. (1929): I sarcofagi cristiani antichi. Roma.

\section{BIBLIOGRAFÍA}

BEJAOUI, F. (1997): Céramique et Religion Chrétienne: les thèmes bibliques sur la sigillée africaine. Tunis.

BENDALA, M. (1971): "Dos fragmentos de sarcófagos paleocristianos", Habis 2: 273-282.

BISCONTI, F. (2004): "I sarcofagi del paradiso", en F. Bisconti y H. Brandenburg (dirs.), Sarcofagi tardoantichi, paleocristiani e altomedievali: 53-74. Città del Vaticano. 
BRANDENBURG, H. (2002): "Das Ende der antiken Sarkophagkunst in Rom. Pagane und christliche Sarkophage im 4. Jhr", en G. Koch (coord.), Akten Symposium Frühchristliche Sarkophage (Marburg 1999): 19-39. Mainz.

CAILLET, J.P. (1993): "Les sarcophages chrétiens en Provence (III"-Ve s.)", Antiquité Tardive 1: 127-146.

DRESKEN-WEILAND, J. (2004): "Ricerche sui committenti e destinatari dei sarcophagi paleocristiani a Roma", en F. Bisconti y H. Brandenburg (dirs.), Sarcofagi tardoantichi, paleocristiani e altomedievali: 149-153. Città del Vaticano.

EICHNER, K. (1981): "Die Produktionsmethoden der stadtrömischen. Sarkophagfabrik in der Blütezeit unter Konstantin", Jarbuch für Antike und Christentum 24: 85-113.

EWALD, B. (2003): "Sarcophagi and Senators: The Social History of Roman Funerary Art and its Limits", Journal of Roman Archaeology, 16: 561-571.

FITA, F. (1887): "Sarcófago cristiano de Écija", Boletín de la Real Academia de la Historia X: 267.

FICKER, J. (1887): "Die christlichen Sarkophage Spaniens", Römische Mitteilungen IV: 87-88.

GARCÍA GARCÍA, M.A. (2004): "La reutilización y destrucción de los sarcófagos romanos de Baetica durante la Edad Media", ROMVLA 3: 239-256.

GUYON, J. (2001): "La rapide floraison d'un décor chrétien sur les sarcophages", en J. Guyon, M. Heijmans (dirs.), D'un monde à l'autre. Naissance d'une Chrétienté en Provence IV $V^{e}-V I^{e}$ siècle: 50-55. Arles.

IMMERZEEL, M. (1996): De sarcofaagindustrie rond 400. Het westelijke Middellandse Zeegebied. Leyden.

IMMERZEEL, M. y JONGSTE, P. (1993): "Import and Local Production of Early Christian Sarcophagi in France", Boreas 16: 135-148.

KAJANTO, I. (1962): "On the problem of names of humility", Arctos 3: 45-53.

KOCH, G. y SICHTERMANN, H. (1982): Römische Sarkophage. München.

KOCH, G. (2000): Frühchristliche Sarkophage. München.

LAWRENCE, M. (1927) : “City-gate Sarcophagi”, The Art Bulletin 10, 1: 1-45.

MAZZEI, B. (2004) : "A proposito del sarcofago di Bethesda delle catacombe di Pretestato", en F. Bisconti y H. Brandenburg (dirs.), Sarcofagi tardoantichi, paleocristiani e altomedievali: 111130. Città del Vaticano.

NICOLETTI, A. (1981): I sarcofagi di Bethesda. Milano.

PENSABENE, P. (1993): “Il reimpiego nell'età costantiniana a Roma”, en G. Bonamente y F. Fusco (eds.), Costantino il Grande dall'antichità all'umanesimo. Colloquio sul cristianesimo nel mondo antico: 749768. Macerata.

RODÀ, I. (2001): "Producción, materiales y circulación de sarcófagos en el Imperio Romano", en J.M. Noguera, E. Conde, El sarcófago romano. Contribuciones al estudio de su tipología, iconografia y centros de producción: 51-78. Murcia.

RODRÍGUEZ OLIVA, P. (2002): "Un sarcófago del Museo Arqueológico Provincial de Sevilla y su relación con otros sarcófagos hispanos de estrígiles", Baetica 24: 275-298.

RUSSELL, B. (2011): “The Roman Sarcophagus "Industry": a Reconsideration", en J. Elsner, J. Huskinson (eds.), Life, death and representation. Some new work on roman sarcophagi : 119-148. Göttingen.

SALES Y FERRÉ, M. (1887): Estudios Arqueológicos e Históricos. Madrid.

SÁNCHEZ VELASCO, J. (2008) : “El sarcófago tardoantiguo del Camino Viejo de Almodóvar (o de los límites de la iconografía)", Spal 17: 335-348. http:// dx.doi.org/10.12795/spal.2008.i17.16

SIMON, M. (1938), "Sur l'origine des sarcophages chrétiens du type Bethesda", Mélanges d'Archeologie et d'Histoire 55 : 201-223.

SOTOMAYOR, M. (1973): Datos históricos sobre los sarcófagos romano cristianos de España. Granada.

SOTOMAYOR, M. (1975): Sarcófagos romano cristianos de España: estudio iconográfico. Granada.

VAN KEUREN, F.; ATTANASIO, D.; HERRMANN, J.J.; NORMAN, J. y PETER, L. (2011): "Multimethod Analyses of Roman Sarcophagi at the $\mathrm{Mu}-$ seo Nazionale Romano, Rome", en J. Elsner y J. Huskinson (eds.), Life, death and representation. Some new work on roman sarcophagi: 149-188. Göttingen.

WARD PERKINS, J.B. (1978): "The role of the craftsmanship in the formation of early christian art", en Atti del IX Congresso Internazionale di Archeologia Cristiana. Roma 21-27 Settembre 1975, I: 637653. Città del Vaticano. 